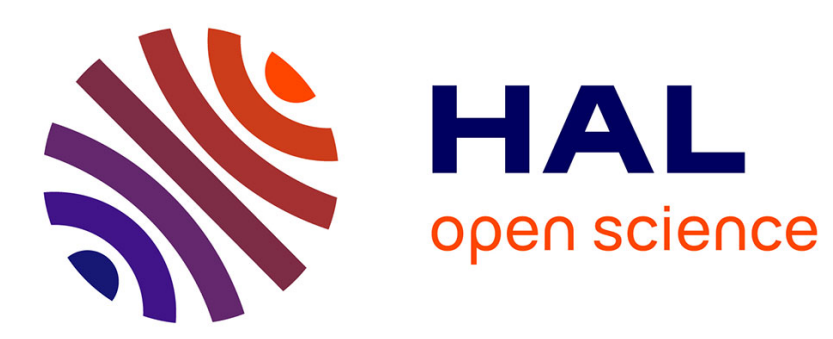

\title{
Les pesticides organochlorés dans les laits de la Haute-Vienne au printemps 1974
}

\author{
J.A. Nicolas, P. Carassus, S. Allaire
}

\section{To cite this version:}

J.A. Nicolas, P. Carassus, S. Allaire. Les pesticides organochlorés dans les laits de la Haute-Vienne au printemps 1974. Le Lait, 1975, 55 (548), pp.544-551. hal-00928704

\section{HAL Id: hal-00928704 \\ https://hal.science/hal-00928704}

Submitted on 1 Jan 1975

HAL is a multi-disciplinary open access archive for the deposit and dissemination of scientific research documents, whether they are published or not. The documents may come from teaching and research institutions in France or abroad, or from public or private research centers.
L'archive ouverte pluridisciplinaire HAL, est destinée au dépôt et à la diffusion de documents scientifiques de niveau recherche, publiés ou non, émanant des établissements d'enseignement et de recherche français ou étrangers, des laboratoires publics ou privés. 


\title{
Les pesticides organochlorés dans les laits de la Haute-Vienne au printemps 1974
}

\author{
par \\ J.A. NICOLAS* \\ P. CARASSUS** et Mme S. ALLAIRE
}

\section{I. - INTRODUCTION}

Depuis que se manifeste une réelle sensibilisation de l'opinion publique aux phénomènes de pollution, considérés, à raison, comme un danger potentiel de notre civilisation, la présence de résidus de pesticides constitue un problème qui préoccupe considérablement les hygiénistes.

Ce problème s'avère particulièrement délicat en ce qui concerne le lait qui constitue la nourriture de base de l'homme et qui demeure l'aliment essentiel des enfants en bas âge.

C'est la commercialisation du D.D.T. à la fin de la dernière guerre et son emploi inconsidéré qui ont posé le problème de la pollution du lait et des produits laitiers par les pesticides.

De nos jours une faible partie de la population suffit à produire les quantités de denrées alimentaires nécessaires à l'alimentation de l'ensemble des habitants grâce aux perfectionnements des techniques agricoles : fertilisation, irrigation des sols, mécanisation de l'agriculture, sélection végétale et animale, lutte contre les maladies et les parasites des plantes et des animaux.

Parmi ces moyens de lutte, les pesticides (de l'anglais pest : fléau) occupent une place prépondérante. En l'état actuel de nos connaissances, il est impensable de se priver de ces produits qui empêchent une réduction considérable des rendements agricoles laquelle impliquerait rapidement la réapparition de la disette et même de la famine. Mais en contrepartie de cet avantage ils présentent un grave inconvénient : il s'agit de substances toxiques dont

\footnotetext{
* Directeur du Laboratoire départemental d'analyses et de recherches.

** Responsable du Service de Biochimie.
} 
certaines risquent de laisser dans l'aliment des résidus, à des doses certes infimes, mais dont on ne peut préciser l'incidence à long terme sur la santé de l'homme.

Les pesticides organo-chlorés ( $\mathrm{HCH}$, lindane, heptachlore, aldrine, D.D.T. et leurs dérivés) ont la double propriété d'être rémanents et liposolubles ce qui entraîne par suite d'ingestions répétées une accumulation dans l'organisme et notamment au niveau des cellules adipeuses.

En conséquence de ce risque méconnu, est apparu dans les pays développés une réglementation très stricte fixant le taux de chaque organo-chloré dans la matière grasse du lait. En France une telle réglementation est inexistante et ce n'est qu'un problème économique (le refoulement des fromages français par les U.S.A.) qui a incité le législateur à prendre des mesures pour que de tels incidents ne se renouvellent plus.

Avant 1969 la cause principale de la pollution du lait par les pesticides était le traitement des étables et des locaux de traite par des produits destinés à détruire la mouche et dont le principe actif était un insecticide organo-chloré $(\mathrm{HCH}$, aldrine, dieldrine) et son passage dans la matière grasse du lait des vaches s'expliquait par le léchage des murs ainsi traités.

L'interdiction de l'emploi de ces produits pour la désinfection des locaux d'élevage le 11 octobre 1969 a fait baisser spectaculairement cette contamination.

Aujourd'hui la source essentielle de pollution du lait par les résidus est l'alimentation du bétail par les céréales tourteaux et concentrés qui tendent à remplacer les fourrages traditionnels ainsi que par les pulpes de betteraves rétrocédées ou par des pulpes sèches importées et souvent contaminées en heptachlore dont l'emploi en agriculture est interdit par l'arrêté du 25 octobre 1972.

Il peut également se produire localement des emplois frauduleux de produits interdits (gardés en stocks).

A ces différentes causes de pollution s'ajoutent des pratiques extérieures au producteur de lait notamment les traitements vétérinaires avec des produits à base de lindane.

\section{II. - BUT DE L'ETUDE}

A la suite des interdictions successives prises par le législateur supprimant les causes principales de pollution il nous a paru intéressant du fait de la rémanence des organo-chlorés ( 3 ans pour l'aldrine, 6 ans pour le lindane) de déterminer le taux actuel de la pollution des laits de la Haute-Vienne. 


\section{III. - ECHANTILLONNAGE}

Nous avons sélectionné les producteurs qui au début du mois d'avril 1974 assuraient une production quotidienne minimale de 1501 de lait. Leur nombre s'élève à 300 soit un échantillon représentatif des 1000 producteurs du département.

\section{IV. - METHODE ANALYTIQUE}

Nous avons adopté la méthode de Demaimay, Lavoue, Feuillat.

\section{Réactifs}

- Ether éthylique Merck p.a.

- Acétone Merck p.a.

- Ether de Pétrole Merck (traité selon technique du J.O. du 31-12-68, page 6261 ).

Tous les solvants sont distillés dans des appareils en verre en éliminant la tête et la queue de la distillation.

Après concentration chaque lot est testé au chromatographe pour s'assurer de l'absence d'impuretés :

- Sulfate de sodium anhydre pour analyses Merck.

- Laine de verre.

- Pesticides standards purs (supelco).

\section{Matériel}

- Chromatographe en phase gazeuse Packard modèle 803 équipé d'un détecteur à capture d'électrons utilisant le Ni 63 comme source radioactive.

Colonne en verre 6 pieds garnie de DC 200 à $10 \mathrm{p} .100$ sur Anakrom AB S ( 80 - 90 Mesh) précolonne $(15 \mathrm{~cm})$ gaine de Réoplex 400 à 10 p. 100 sur chromosorb W. AW. (80 - 100 Mesh).

- Pulseur Packard : période 100/s, amplitude $50 \mathrm{~V}$.

- Enregistreur Honeywell $1 \mathrm{mV}$ pleine échelle :

- Isothermie à $160^{\circ} \mathrm{C}$.

- Injecteur à $190^{\circ} \mathrm{C}$.

- Détecteur à $265^{\circ} \mathrm{C}$.

- Gaz vecteur Argon à $50 \mathrm{ml} / \mathrm{mn}$.

\section{Mode opératoire}

- Centrifuger $100 \mathrm{ml}$ de lait à $7000 \mathrm{t} / \mathrm{mn}$ pendant $15 \mathrm{mn}$ et éliminer la phase aqueuse. 
- Récupérer la matière grasse par $100 \mathrm{ml}$ du mélange acétone, éther éthylique, éther de pétrole (80.60.60).

- Ajouter quelques gouttes d'oxalate $\mathrm{Na}$ à 5 p. 100 (anti-émulsion) et $300 \mathrm{ml}$ d'eau salée à $20 \mathrm{~g} / 1$. Après agitation laisser décanter et éliminer la phase aqueuse qui entraîne les impuretés hydrosolubles.

- Dessécher la phase éthérée sur une colonne de $\mathrm{SO}_{4} \mathrm{Na}_{2}$ anhydre.

- Evaporer les solvants sous vide léger.

- Récupérer la matière grasse purifiée dans un tube préalablement taré et stocker au congélateur jusqu'au moment de l'analyse.

- Au moment de l'analyse un étalon interne est ajouté à la matière grasse et après une bonne homogénéisation une fraction est injectée au chromatographe.

- Les courbes d'étalonnage étant réalisées en fonction de cet étalon interne (fig. 1) la précision de la quantité d'échantillon infectée n'est pas à considérer.

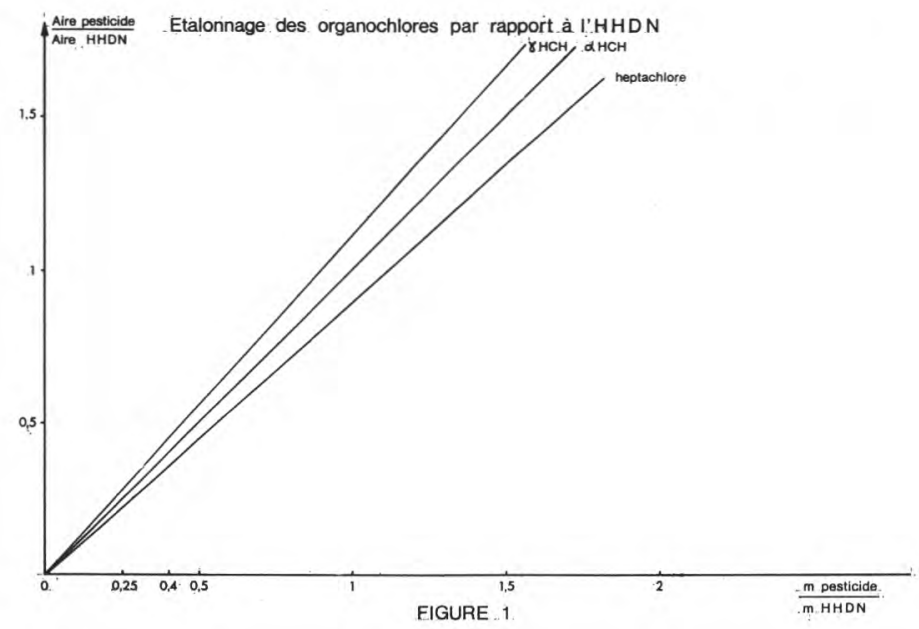

fig. 1

\section{V. - RESULTATS ET INTERPRETATION}

Bien qu'une réglementation soit en cours d'élaboration au sein de la C.E.E. il n'existe actuellement en France aucune norme concernant les taux des pesticides organo-chlorés dans le lait et ses dérivés. 
Nous avons donc adopté comme seuil de comparaison les recommandations du Codex OMS/FAO qui sont une synthèse des tolérances administratives en vigueur, soit :

- 0,2 $\mathrm{mg} / \mathrm{kg}$ de M.G. pour l' $\alpha \mathrm{HCH}$.

- 0,1 $\mathrm{mg} / \mathrm{kg}$ de M.G. pour le $\gamma \mathrm{HCH}$ (lindane).

- 0,125 mg/kg de M.G. pour l'heptachlore.

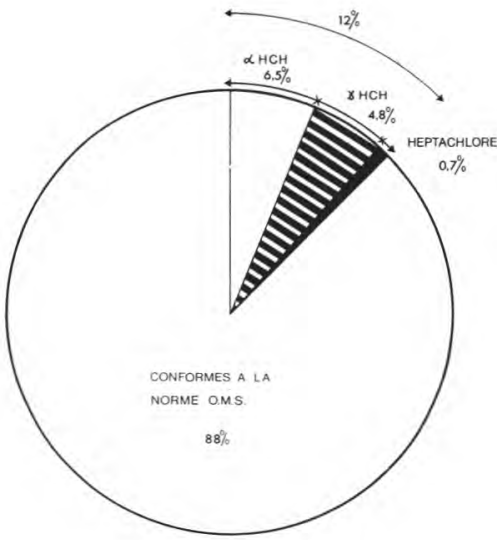

fig. 2



fig. 3

Sur 305 échantillons prélevés 292 ont pu être exploités et il ressort que 35 des laits analysés soit 12 p. 100 sont au-dessus de la norme OMS (fig. 2). Ces 12 p. 100 se répartissent en :

- 6,5 p. 100 pour des laits contaminés par l' $\alpha \mathrm{HCH}$.

- 4,8 p. 100 pour des laits contaminés par le $\gamma \mathrm{HCH}$.

- 0,7 p. 100 pour des laits contaminés par l'heptachlore.

C'est l'aHCH qui représente la majorité des laits pollués avec 54,2 p. 100 suivi du $\gamma \mathrm{HCH}$ (lindane) avec 40 p. 100 puis de l'heptachlore avec seulement 5,8 p.100 (fig. 3).

La moyenne de la pollution pour l' $\alpha \mathrm{HCH}$ est de $0,1 \mathrm{mg} / \mathrm{kg}$ de M.G. soit la moitié du taux préconisé par l'O.M.S. et pour le $\gamma \mathrm{HCH}$ alors que la moyenne nationale s'élève à $0,08 \mathrm{mg} / \mathrm{kg}$ pour cet isomère (fig. 4 et 5 ).

Il est à noter que 2 échantillons ne contenaient pas d' $\alpha \mathrm{HCH}$ et 58 ne contenaient pas de lindane, le seuil de sensibilité de la méthode de dosage utilisé étant de $0,01 \mathrm{mg} / \mathrm{kg}$ de M.G.

Il est également important de souligner le faible nombre d'échantillons contaminés par l'heptachlore mais aussi l'absence d'aldrine, de dieldrine et de D.D.T. 


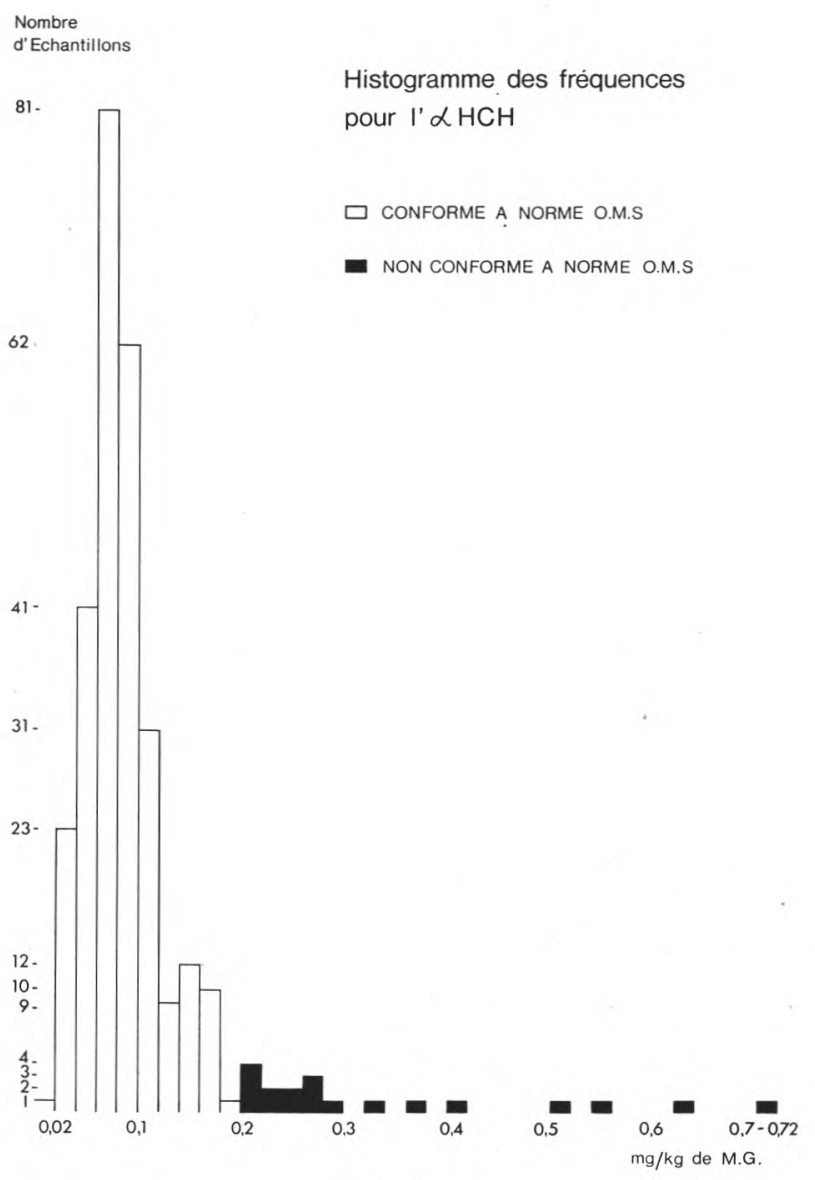

fig. 4

De plus de ces différents résultats il ressort que les laits de la Haute-Vienne sont dans leur très grande majorité, très satisfaisants en ce qui concerne leur contamination par les pesticides organochlorés puisque 88 p. 100 sont conformes aux normes O.M.S. Cependant la fraction non conforme (12 p. 100) peut être sensiblement réduite si la législation interdisant l'utilisation des organo-chlorés en agriculture est respectée. Il y a là un travail d'information des producteurs laitiers car la répartition géographique des échantillons pollués laisse apparaître qu'il s'agit de cas isolés. 


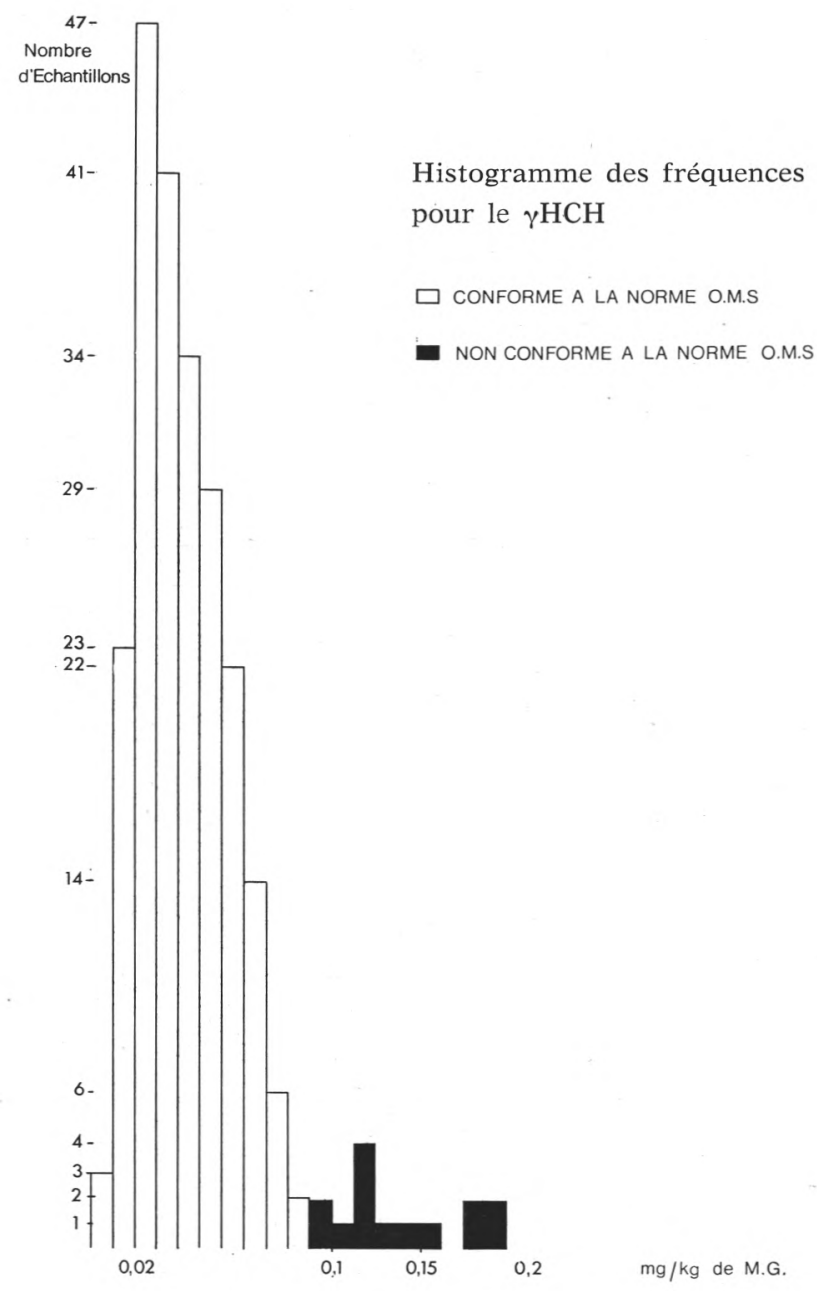

fig. 5

\section{Remerciements}

Nous remercions Mlle M.C. Nicolas, directrice du Laboratoire interprofessionnel laitier de la Haute-Vienne, qui nous a fourni les échantillons analysés.

Nos remerciements s'adressent également à $M$. Casteignau de la Faculté des Sciences de Limoges pour ses conseils éclairés. 


\section{S u m m a r y}

Organochlorinated being prohibited in Agriculture, the authors were looking for the rate of polluted milk with these pesticides in Haute-Vienne.

In Spring 1974, 12 p. 100 of 300 samples analysed contained more pesticides than tolerated by O.M.S. norms, with a majority of BHC isomers (11,3 p. 100).

Key-Words : cow-milk, organochlorinated pesticides.

\section{Bibliographie}

Demaimay (M.), Lavoue (G.), Feuillat (M.) (1972). - Essai de dosage qualitatif et quantitatif de quelques organo-chlorés dans les produits laitiers par l'utilisation d'une précolonne en C.G.L. Le Lait, n 511-512, p. 43.

Eidelman (M.) (1962). - Détermination of micro quantities of chlorinated organic pesticide residus. Journal of A.O.A.C., vol. 45, $\mathrm{n}^{\circ} 3$.

FRIES (G. F.) (1970). - Organochlorine pesticides and the dairy industry. Journal of Dairy Sci., vol. 53, $\mathrm{n}^{\circ} 3$.

Hasloet (M.) (1970). - Les résidus de pesticides dans les produits laitiers. B.T.I., $\mathrm{n}^{\circ} 252,59$.

Luquet (F.), Goursaud (J. G.), Casalis (J.) (1974). - Les résidus de pesticides organochlorés dans les laits animaux et humains. Ann. Fals. Exp. Chim., 67, n 716 , p. $217-239$.

Luquet (F.) (1973). - Thèse : Contribution à la connaissance de la pollution des laits en R.P.O. Université de Caen.

Mahieu (H.) (1974). - Causes et remèdes de la contamination du lait par les pesticides organochlorés. Prod. Agr. Français, mai.

Smart (N. A.), Hill (A. R. C.) (1972). - Residues of somme chlorimated hydrocarbon pesticides in milk products after their addition to the fad of cows. Pesticide Science, 3415-3419.

Tuinstra (L.G.M. Th.), Roos (J. B.) (1970). - Residual content of organo-chlorure insecticides. Neth Milk and Dairy J., 24, 65-78.

Le point de l'évolution des résidus de pesticides dans le lait. Comm. de l'Agr., $1974, \mathrm{n}^{\circ} 88$, septembre. 